\title{
Mid-upper arm circumference (MUAC): new applications for an old measure
}

\author{
Professor John J Reilly \\ University of Strathclyde, Graham Hills Building, Glasgow G1 1XQ, UK
}

Mid-upper arm circumference (MUAC) has a long history as a simple and valuable anthropometric marker of under-nutrition, of particular value in resource-poor settings ${ }^{1}$. Traditionally, low MUAC has been seen as a proxy for low fat-free mass ${ }^{1}$, necessary because accurate measurement of fatfree mass in children is usually impractical in both clinical settings and resource-poor settings.

An emerging body of evidence suggests that MUAC may have value beyond its traditional application in the assessment of under-nutrition. The paper by Whitfield and colleagues in this issue of the journal ${ }^{2}$ notes that accurate measurement of body weight is often not possible in resource-poor settings, and this is important clinically because it substantially increases the risk of inaccurate drug dosage (where dosage is dependent on body weight). Whitfield et $\mathrm{al}^{2}$ describe a study which suggests that MUAC, when combined with other anthropometric measures, could be a useful and accurate way of estimating body weight in children.

In a sample of 775 predominantly HIV positive children and adolescents (from 18 months- 12 years of age) from Botswana, Whitfield et al made a range of anthropometric measures (height or length, ulnar and tibial lengths, MUAC, and triceps skinfold, body weight). They developed prediction equations for body weight in a randomly selected half of the sample, then tested the accuracy of these prediction equations in the other half. They found that body weight could be estimated with high accuracy using a combination of MUAC and height (or length), MUAC and ulnar length, or MUAC and tibial length, all measured with a simple measuring tape. Whitfield et al also estimated body weight using a range of alternative equations which have been used or recommended, most of which are based on samples from high-income countries: errors in estimation of weight were far higher than with the newly developed MUAC approach, and typically unacceptably high. The MUAC and ulnar and tibial length measures made in the study by Whitfield et al $^{2}$ all had high precision (low inter-observer error) when made by the trained observers in the study, as expected ${ }^{1}$. In summary, MUAC appears to be a promising option for the estimation of body weight where this cannot be measured or cannot be measured accurately-this could improve the accuracy of drug dosage, as well as making weight-based measures of nutritional status (e.g. BMI for age) possible in some resourcepoor settings. A few caveats are worth considering. First, as noted by the authors, prediction equations have to be converted to simpler calculation-free methods if they are to be used accurately and widely in clinical practice, since even apparently simple calculations to assess growth or nutritional status can produce large errors when used clinically ${ }^{3}$ : the authors provide nomograms for this purpose ${ }^{2}$. Second, generalisability of study findings is important. Prediction equations of the kind developed by Whitfield et $\mathrm{al}^{2}$ are notoriously population-specific, and external cross-validation in different populations would be important, along with evidence that the nomograms provided were practical and accurate, and inter-observer agreement high. Finally, when body weight cannot be measured then a proxy is clearly essential, but the issue of knowing when body weight 
measurement is inaccurate (e.g. when scales are available but inaccurate) and when MUAC might be preferable, is more complicated than it might seem.

Another potentially valuable application of MUAC measurement in resource-poor settings has emerged recently, with new evidence that MUAC can provide high accuracy for the assessment of obesity in children and adolescents ${ }^{5}$. A number of studies from low-, middle, and high-income countries have shown that high MUAC has high diagnostic accuracy (sensitivity, specificity, predictive values) for the identification of overweight and obesity (as defined by BMI for age ${ }^{4}$ ) and for the identification of overfatness (as measured by body composition techniques ${ }^{5}$ ). While MUAC has been seen as a marker of fat-free mass ${ }^{1}$, children and adolescents with high MUAC are highly likely to have excess body fat mass, high weight for age, and high BMI for age $\mathrm{e}^{4,5}$. Since MUAC measurement is already well established for the assessment of under-nutrition, it should be relatively easy to extend its use to the assessment of over-nutrition, an increasingly important application given the continuing childhood obesity pandemic. Measurement of MUAC could therefore provide a novel, simple, and accurate means of identifying child and adolescent obesity in clinical settings, and of population surveillance of obesity in settings where more complicated measures were not practical. One caveat over the use of MUAC for assessment of over-nutrition is that it is not yet clear what the optimal MUAC 'cutpoints' are for this purpose, and the extent to which these cut-points might be age- specific or population-specific is not completely clear ${ }^{4,5}$.

In summary, an emerging body of evidence suggests that measurement of MUAC may have substantial additional value, in both clinical practice and public health, beyond its traditional role as a means of assessing under-nutrition. Specifically, MUAC might provide a means of estimating body weight, and a means of identifying obese children, when body weight measurement is not possible, or is of doubtful accuracy. Given the established importance of child body weight status to current and future health and human capital, and in the era of sustainable development goals and global actions on obesity and obesity- related NCDs, it is an indictment of global wealth distribution that there are so many settings where body weight cannot be measured, or cannot be measured accurately. Nonetheless, given the current situation, the use of MUAC for estimation of body weight, and for assessment of obesity, is clearly promising, and for some purposes may even prove to be transformative.

929 words

References Cited

1. RS Gibson. Assessment of fat-free mass. Chapter 11, pp 200-205 In: Principles of Nutritional Assessment, Oxford University Press, $1^{\text {st }}$ ed, 1990.

2. KC Whitfield, R Wozniak, M Pradinuk et al. Anthropometric measures are simple and accurate weight prediction proxies in resource poor settings with a high HIV prevalence. Arch Dis Child, In press---this issue.

3. Poustie V, Watling RM, Ashby D, Smyth RL. Reliability of percentage ideal weight for height.. Arch Dis Child 2000; 83: 183-184. 
4. Chaput JP, Katzmarzyk PT, Barnes JD. Mid-upper arm circumference as a screening tool for identifying children with obesity: a 12 country study. Pediatr Obes, In press.

5. Craig E, Bland RM, Ndirangu J, Reilly JJ. Use of mid-upper arm circumference for assessing overweight and overfatness in children and adolescents. Arch Dis Child 2014; 99: 763-766. 